\title{
Monitoramento de informação em mídias sociais: o e-Monitor Dengue
}

\author{
Social media monitoring: The \\ Dengue e-Monitor
}

Michele Nacif ANTUNES ${ }^{1}$

Cícera Henrique da SILVA²

Maria Cristina Soares GUIMARÃES²

Marcelo Henrique Leoni RABAÇO ${ }^{3}$

\section{Resumo}

Este artigo apresenta os resultados preliminares da pesquisa "Monitoramento de informação sobre doenças negligenciadas: 0 e-Monitor Dengue". O e-Monitor Dengue é um sistema de monitoramento de informação na Internet feito por meio de um mecanismo robô, software ou agente inteligente que vasculha os sites sobre dengue disponíveis na Internet. Resultados de pesquisas anteriores indicaram novas perspectivas para o monitoramento, principalmente, por meio das mídias sociais. Dentre as mídias sociais, considera-se que o Twitter pode desempenhar um papel na gestão da informação ao permitir identificar usuários que podem atuar como filtro de informação, sendo possível acessar diretamente a informação mais relevante para uma determinada área de interesse. Assim, a partir do monitoramento do Twitter, a primeira pergunta a ser respondida no âmbito da pesquisa em andamento foi "Quem fala sobre dengue?". Inicialmente, foi possível identificar a relação dos atores/perfis localizados na cidade do Rio de Janeiro e Niterói com maior ocorrência do termo "dengue" em suas mensagens. Os perfis foram cadastrados no sistema de monitoramento e são monitorados constantemente. Outra pergunta a ser respondida foi "Quando se fala de dengue?". Observa-se que o número de twetts acompanha o crescimento do número de casos de dengue. São indícios de que há uma relação entre os rumores sobre dengue e o aumento de número de casos notificados. Evidencia-se, desta forma, que realizar o monitoramento nas mídias sociais durante o período de epidemia e fazer sua relação com a situação epidemiológica da dengue pode ser uma estratégia importante para as autoridades em vigilância epidemiológica.

Palavras-chave: Dengue. Mídias sociais. Monitoramento de informação. Redes sociais. Vigilância em saúde.

\section{Abstract}

The aim of the article is to discuss the preliminary results of the research "Information monitoring on neglected diseases: the Dengue e-Monitor". The Dengue e-Monitor is an information monitoring system on the Internet. Monitoring is achieved through robot mechanisms, softwares or intelligent agents that search for websites about dengue available on the Internet. Results of previous studies have indicated new perspectives for information monitoring mainly through social media. Among social media, Twitter is considered to play a key role in information management by allowing the identification of users who may act as filters of information, thus enabling

\footnotetext{
1 Fundação Oswaldo Cruz, Instituto de Comunicação e Informação Científica e Tecnológica em Saúde, Laboratório de Informação Científica e Tecnológica em Saúde, Programa de Inovação Tecnológica. Av. Brasil, 4365, Pavilhão Haity Moussatché, Manguinhos, 21045-360, Rio de Janeiro, RJ, Brasil. Correspondência para/Correspondenceto: M.N. ANTUNES.E-mail:<mnacif@icict.fiocruz.br>.

2 Fundação Oswaldo Cruz, Instituto de Comunicação e Informação Científica e Tecnológica em Saúde, Programa de Pós-Graduação em Informação e Comunicação em Saúde. Rio de Janeiro, RJ, Brasil.

3 Fundação Oswaldo Cruz, Instituto de Comunicação e Informação Científica e Tecnológica em Saúde, Centro de Tecnologia de Informação e Comunicação. Rio de Janeiro, RJ, Brasil.

Recebido em 25/10/2012, reapresentado em 23/7/2013 e aceito para publicação em 19/8/2013.
} 
direct access to the most relevant information in a particular area of interest. Therefore, by monitoring Twitter, the first question to be answered was "Who talks about dengue?". Initially, it was possible to identify the relationship between the actors/profiles located in the city of Rio de Janeiro and Niterói with the highest occurrence of the word "dengue" in their posts. The profiles were then registered in the monitoring system and are constantly monitored. The second question to be answered was "When is dengue mentioned?" With this monitoring system, we expect to gain a better understanding of how social media can be used to strengthen the relationship between science and society. It was found that the number of twetts accompanied the growth in the number of dengue cases. Indications are that there is a relationship between rumors about dengue and the increase in the number of cases reported. It is evident, therefore, that monitoring social media during an epidemic and relating the findings to the epidemiological situation of dengue can be an important strategy for the epidemiological surveillance system.

Keywords: Dengue. Social networks. Information monitoring. Social media. Health surveillance.

\section{Introdução}

Este artigo apresenta os resultados preliminares da pesquisa "Monitoramento de informação sobre doenças negligenciadas: o e-Monitor Dengue". O e-Monitor Dengue é um sistema de monitoramento de informação na Internet, construído com a finalidade de auxiliar na vigilância de epidemias de dengue. O monitoramento é feito por meio de um mecanismo robô, software ou agente inteligente que vasculha os sites sobre dengue disponíveis na Internet.

A Organização Mundial da Saúde (OMS) estima que 2,5 bilhões de pessoas estão sob o risco de contrair dengue e que ocorram anualmente cerca de 50 milhões de casos, dos quais cerca de 550 mil serão hospitalizados e pelo menos 20 mil morrerão em consequência da doença (Organização Mundial da Saúde, 2009). Monitorar o ambiente informacional é, portanto, essencial.

Na perspectiva de monitorar para vigiar e antecipar eventos futuros, insere-se o e-Monitor Dengue como um espaço para a circulação de informação e conhecimento de caráter estratégico para a vigilância em saúde e tomada de decisão.

Nessa mesma linha de pesquisa, foi desenvolvido, em 2007, o projeto "Monitoramento de informação na sociedade de risco: o caso da influenza aviária" (Silva, 2007). A pesquisa teve como objetivo o desenvolvimento de um sistema de monitoramento de informação sobre pandemia de influenza. O monitoramento foi realizado por um programa-robô especialmente desenvolvido para tal finalidade. Ao final da pesquisa, foi disponibilizado o e-Monitor Influenza <http://157.86.8.16/emonitor/>, um espaço virtual de monitoramento e agregador de informação oficial, de qualidade e atualizada, capaz de possibilitar ao tomador de decisão uma visão sistêmica e mais integradora das competências e do status das ações e esforços nacionais na prevenção da pandemia (Guimarães et al., 2008). Ao observar o fluxo do monitoramento, foi evidenciado que o ciclo da geração da informação é iniciado pela notícia oficiosa, aquela presente na grande mídia (atualidade) até a avaliação e divulgação nos sítios oficiais como evidência de saúde (certificação). Ao capturar dados que frequentemente escapam às fontes de informação oficiais, abriram-se novas perspectivas para o monitoramento, principalmente, por meio das mídias sociais.

É então sob essa nova perspectiva de monitoramento que se intenciona responder ao longo da pesquisa, ainda em curso, à seguinte questão: de que forma as mídias sociais podem ser mobilizadas para fortalecer a relação ciência-sociedade?

\section{Monitoramento de mídias sociais: o Twitter}

A definição do termo mídias sociais ainda está em evolução, sem uma definição universalmente aceita. De acordo com Sterne (2011), a Internet sempre atuou como uma mídia social, pois foi o primeiro canal de comunicação de "muitos-para-muitos". Desta forma, ela é única porque sempre esteve relacionada à capacidade de uma pessoa se comunicar com o resto do mundo. $\mathrm{O}$ autor afirma ainda que há seis grandes categorias de mídias sociais: os fóruns e quadros de mensagens, sites de crítica e opinião, marcadores sociais, compartilhamento de mídia, blogs, microblogs e redes sociais.

As redes sociais implicam uma maior interatividade entre participantes, pois permitem a criação de um grupo aberto ou fechado voltado à comunicação, colaboração e contato pessoal, são comunidades semiabertas para conexão online. Nestes ambientes, os 
espaços são desenvolvidos especialmente para troca de informação e experiências, como o Facebook (Keckley, 2010).

No setor privado, as mídias sociais ganharam destaque por permitirem a comunicação entre as organizações e seus clientes e possibilitaram a troca de opiniões sobre serviços e produtos entre clientes. Surpreendentemente, as tendências das redes sociais se estenderam à arena de cuidados de saúde, uma vez que aqueles que procuram informação sobre saúde online começaram a disseminar suas experiências e conhecimento no coletivo da rede mundial de computadores (Scanfeld et al., 2010).

Thackeray et al. (2012) afirmam que, no campo da saúde pública, as mídias sociais podem ser utilizadas para informar, educar e capacitar as pessoas sobre os problemas de saúde, para melhorar a velocidade na comunicação durante emergências de saúde ou surtos, para mobilizar a comunidade para parcerias e ações, para facilitar a mudança de comportamento, para coletar dados de vigilância, e para entender percepções sobre questões públicas.

Dentre as mídias sociais, o microblog Twitter.com apresenta uma promissora fonte para o monitoramento de informação em saúde, pela rapidez e volume de mensagens (Keckley, 2010). O Twitter é um serviço de microblog que permite aos seus usuários ler e enviar mensagens de textos com até 140 caracteres, chamados de tweets. A rede de contatos é um sistema que permite seguir usuários e ser seguido por outros usuários sem a necessidade de autorização. Qualquer usuário pode responder ou encaminhar, ou seja, retwitar qualquer tweet e iniciar um debate público ou conversa (Torrente et al., 2012). Além disso, de acordo com Akshay et al. (2007), ao mapear a latitude e longitude dos usuários, pode-se extrair também o local de origem e o destino de cada usuário. Desta forma, em muitos casos, o conteúdo dos tweets pode ser analisado pelas autoridades de saúde em tempo real e pode identificar precocemente preocupações do público.

No estudo "Pandemics in the age of twitter: content analysis of tweets during the 2009 H1N1 outbreak", Eysenbach e Chew (2010) utilizaram o Twitter para entender as percepções do público sobre uma pandemia de influenza causada pelo subtipo de Influenzavirus A H1N1, que são designados usando um número H (hemaglutinina) e um número N (neuraminidase). De acordo com os autores, o twitter é potencialmente adequado para a mineração de textos e análise longitudinal. Os textos curtos, o compartilhamento dos usuários com os seguidores de seus pensamentos, sentimentos, atividades, contêm grande riqueza de dados sobre opiniões públicas e comportamentos. Para esses autores, além de uma análise quantitativa, a análise do Twitter permite também uma exploração qualitativa das prováveis razões de mudanças repentinas na comunicação, por exemplo, quando uma notícia é amplamente lida e comentada, evidenciando a atenção do público para um determinado tema. Os autores demonstram nesse estudo que o aumento acentuado no Twitter de mensagens com o termo H1N1 estava relacionado com o volume de notícias sobre o anúncio emitido pela OMS da ocorrência de uma pandemia de influenza.

Além disso, de acordo com os autores Vance et al. (2009), o Twitter pode desempenhar um papel importante na gestão da informação. Ele permite identificar indivíduos, instituições e periódicos e, desta forma, atua como filtro de informação, pois, por meio das mensagens compartilhadas, é possível acessar diretamente a informação mais relevante para uma determinada área de interesse. As agências internacionais como a OMS <@whonews> ou o Centro Controle de Doenças dos Estados Unidos <@CDCgov> utilizam o twitter para divulgar recomendações, surtos de doenças e novas estratégias de prevenção.

Em sintonia com a importância das redes sociais para a saúde no Brasil, há iniciativas recentes do Ministério da Saúde para monitorar informação nas redes sociais sobre dengue por meio do Twitter. O objetivo é utilizar os "rumores" que circulam nas redes sociais para identificar onde os casos de dengue estão surgindo e, assim, preparar uma estratégia de combate (Brasil, 2011). No entanto, não há notícias, até o momento, de alguma estratégia ou de algum dispositivo que possibilite a captura, o tratamento e a disponibilização dessas mensagens de forma estruturada, de maneira a permitir a tomada de decisão pelos gestores.

Desta maneira, as novas ferramentas de monitoramento na Internet diferenciam-se dos sistemas convencionais, pela capacidade de capturar dados que frequen- 
temente escapam às fontes de informação oficiais. Nos últimos anos, a Internet tornou-se parte integrante da vigilância em saúde. Eysenbach (2011) afirma que a coleta e análise dos dados de demandas de informação de saúde na Internet tem um potencial considerável para a vigilância sindrômica. A vigilância sindrômica utiliza os dados relacionados com a saúde que precedem o diagnóstico e indicam a probabilidade suficiente de um surto ou de um caso para justificar uma resposta posterior. De acordo com Yan et al. (2009), a vigilância sindrômica está preocupada com o monitoramento contínuo das fontes de informação relacionadas com a saúde pública e detecção precoce de doenças e, recentemente, tem atraído grande atenção de pesquisadores e gestores. Os sistemas de vigilância sindrômica são adotados para atender à necessidade crítica de prevenção, detecção e gestão de surtos de doenças infecciosas, que ocorrem naturalmente ou são causados por ataques de bioterrorismo.

Enquanto a maioria dos sistemas de vigilância sindrômica utilizam dados convencionais, como já visto anteriormente, emergem novas pesquisas que exploram a Internet como método novo e promissor para a vigilância em saúde pública. Alguns estudos, como Ginsberg et al. (2009) incluem a análise de consultas dos motores de busca para prever surtos de doenças, como, por exemplo, a gripe. O Google Flu Trends <http://www.google.org/ flutrends > utiliza dados agregados de pesquisa do Google para estimar a atividade da gripe até duas semanas mais rápida do que os sistemas tradicionais. Autores como Wang et al. (2009) e Freifeld et al. (2010) exploram o uso de smartphone para avaliar o movimento das populações para a identificação da propagação de epidemias. Como já mencionado anteriormente, o Twitter também se apresenta como potencial para vigilância sindrômica da gripe (Eysenbach \& Chew, 2010).

No que diz respeito à utilização de dados convencionais, Chan et al. (2011) apontam que existe uma variedade de obstáculos que interferem na detecção e notificação de casos de dengue em muitos países endêmicos. De acordo com o autor, os esforços de vigilância voltaram-se para fontes de dados modernas com o uso da Internet, que têm se mostrado eficazes para o monitoramento de doenças semelhantes à gripe. No entanto, há poucos estudos que avaliam os dados de pesquisa na Web para outras doenças, especialmente aquelas de alta morbidade e mortalidade, ou quando não há uma vacina disponível, como no caso da dengue. É sob essa perspectiva que foi desenvolvido o e-Monitor Dengue, no qual o foco do monitoramento em dengue está no seu potencial para a vigilância do ambiente informacional, rastreando rumores de epidemias com maior facilidade e respondendo de forma mais rápida.

\section{O desenvolvimento do e-Monitor Dengue}

Para a Ciência da Informação, monitorar significa observar, checar e atualizar-se em relação à dinâmica de um evento em uma área de interesse, definida para uma finalidade específica (Coates, 1991). Hoffmann (2011) afirma que o monitoramento trabalha como um radar, orientado para detectar e interpretar os sinais do ambiente de uma organização. Para a autora, a abrangência do ambiente informacional ocorre justamente em um contexto atual em que as transformações mais revolucionárias ocorrem nas técnicas e métodos de como lidar com a informação, ou seja, em como acessar, coletar, tratar e analisar a informação. Para Choo e Auster (1993) o monitoramento é a forma essencial de busca de informação por uma organização, com o objetivo de alcançar novo conhecimento que permita a ação.

A prospecção e o monitoramento informacional são os fios condutores para o desenvolvimento do e-Monitor Dengue. O monitoramento informacional é o método ou técnica de observação e acompanhamento constante de dados, informação e conhecimento relevantes ao negócio da organização (Valentim \& Molina, 2004). Por meio da prospecção informacional foi possível estabelecer um mapa inicial de fontes de informação para serem monitoradas.

Inicialmente, as fontes de informação foram classificadas em quatro tipos:

1) Fontes oficiais: instituições nacionais, estaduais, municipais e internacionais, identificadas por meio de documentos e relatórios (OMS, Organização Pan-Americana de Saúde (OPAS), Ministério da Saúde, dentre outras).

2) Fontes de notícias: agências de notícias (BBC News, Reuters, Agência Estado, dentre outras) e grandes jornais diários (O Globo, Folha de São Paulo, entre outras). 
3) Fontes de produção científica: principais periódicos científicos na área de Saúde.

4) Mídias sociais: blogs e o microblog Twitter.

Uma vez definido o mapa de fontes, o programa-robô já desenvolvido anteriormente foi adaptado para o monitoramento de informação a partir do termo "dengue" nas fontes oficiais, fontes de notícias e produção científica. Este programa percorre a Internet recolhendo informações, que são indexadas em uma base de dados e consultadas posteriormente por mecanismos de busca. Assim, o programa-robô é o responsável por monitorar as mudanças nas fontes cadastradas no sistema de monitoramento, emitindo um alerta sempre que um novo conteúdo for publicado. Desta forma, o sistema de monitoramento é capaz de monitorar e capturar informações, por dados pré-definidos (palavras-chave, fontes e links). Nesse caso, o termo definido foi "dengue" e os links cadastrados foram identificados a partir da prospecção informacional. O processamento do conteúdo monitorado consiste em eliminar duplicatas, contar e identificar novos termos, novas fontes e autores. Após essa etapa, o resultado é registrado em uma base de dados, especificamente desenhada para esse fim, possibilitado a emissão de relatórios quantitativos de monitoramento. O conteúdo monitorado é disponibilizado no espaço virtual e-Monitor Dengue de acordo com a tipologia da fonte, como pode ser observado na Figura 1.

Para o monitoramento das mídias sociais, foi realizado um estudo para entender o funcionamento de cada categoria de mídia social, pois cada uma apresenta diferentes características para o monitoramento. Inicialmente, optou-se pelo monitoramento de blogs e do microblog Twitter. Atualmente, o sistema está em fase de adaptação para o monitoramento de outras mídias sociais.

Os primeiros resultados aqui apresentados referem-se ao Twitter, para cujo monitoramento foram consideradas as coordenadas geográficas (latitude e longitude) do Estado do Rio de Janeiro. Assim, foram monitoradas apenas as mensagens postadas pelos usuários localizados no Rio de Janeiro. A base de dados composta por registros do monitoramento do Twitter apresenta um total de 172884 registros obtidos, no período de 9 de abril de 2012 a 15 de setembro de 2012.
A partir do monitoramento do Twitter, a primeira pergunta a ser respondida no âmbito da pesquisa em andamento foi "Quem fala sobre dengue na cidade do Rio de Janeiro e Niterói?". Assim, inicialmente, foi possível identificar a relação dos atores/perfis localizados na cidade do Rio de Janeiro e Niterói com maior ocorrência do termo "dengue" em suas mensagens. Os perfis foram divididos de acordo com o tipo de fonte: oficial, noticiosa, instituição de ensino e profissionais de saúde (Quadro 1). Nota-se que são principalmente as fontes oficiais e as de notícias que divulgaram no Twitter informações sobre dengue no período monitorado. Vale ressaltar que o foco no Twitter está na qualidade e no tipo de conteúdo veiculado por uma fonte específica. Desta forma, é possível seguir, por exemplo, contas de meios de comunicação para receber notícias de jornais, revistas ou canais de televisão, para não ter que acessar os websites de cada um separadamente, ou seguir os perfis de experts de uma determinada área profissional para atualização de pesquisas em tempo real (Santaella \& Lemos, 2010).

Para responder à pergunta "Quando se fala sobre dengue?", foi observada a relação entre os casos notificados por semana epidemiológica na cidade do Rio de Janeiro e os tweets com a ocorrência do termo dengue na mesma localidade e período. Para tal, foi considerado o intervalo entre a $20^{a}$ e $45^{a}$ semana epidemiológica, que corresponde ao período de maio a setembro de 2012, de acordo com a Secretaria Municipal de Saúde e Defesa Civil do Rio de Janeiro (Rio de Janeiro, 2012). No início do período analisado, a cidade do Rio de Janeiro encontrava-se em situação epidêmica e, ao longo do período, os números de casos foram reduzindo, muito em função das ações de combate e mudanças climáticas. Observa-se, de acordo com os casos notificados, que na 20a semana epidemiológica, o Rio de Janeiro já não apresentava uma situação epidêmica de dengue. Seguindo o esperado, o número de tweets também foi mais elevado no início da epidemia. Observa-se que entre a semana 20a e 23a, o número de tweets caiu pela metade e se manteve abaixo de 500 tweets até o fim do período analisado. Assim, quando o Rio sai da situação de epidemia, o número de tweets também reduz, porém mesmo que num nível mais baixo, o assunto não deixou de ser comentado no microblog, ou seja, não houve em nenhuma das semanas epidemiológicas analisadas, zero tweets sobre o tema (Figura 2). 


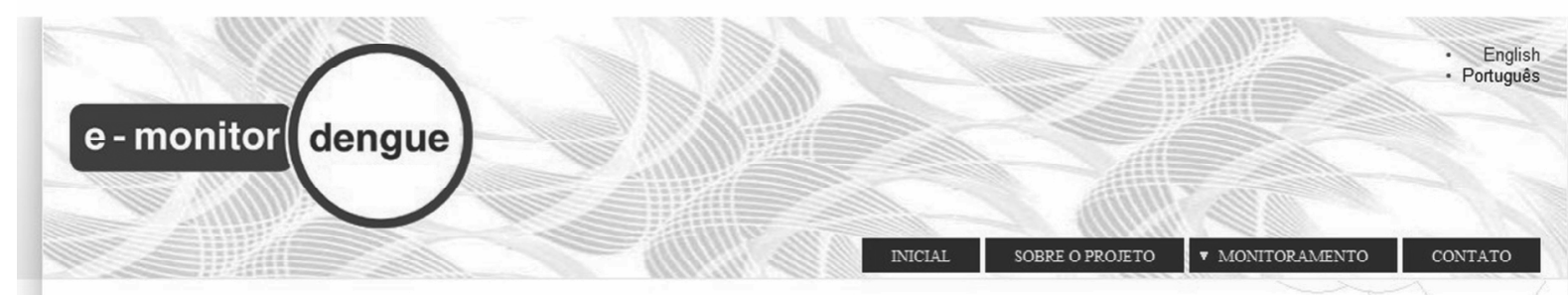

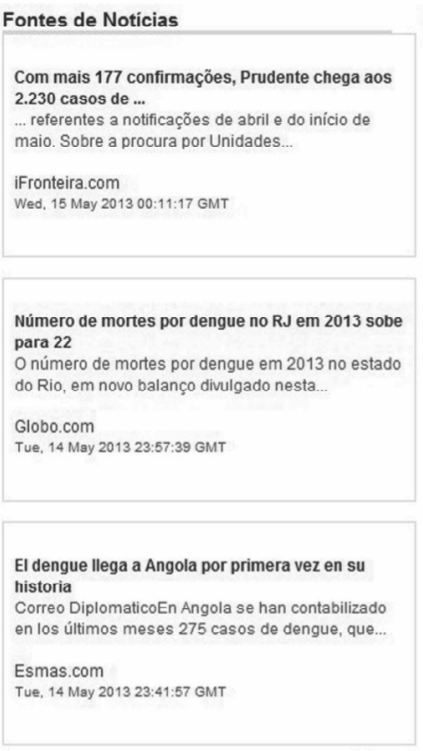

Mais

\section{Blogs}

A novel way to attack malaria by making the mosquitoes resistant to ...

The challenge was to turn a temporary infection into .

Madsen Pirle

Sun. 12 May 20130903.47 GMT

\section{Facebook}

Tem que cuidar direitinho das plantas de casa, viu?

\#combatadengue
Tem que cuidar direitinho das plantas de casa, viu? \#combatadengue

Mural de Combata a Dengue no Facebook Tue, 14 May 2013 23:53:10 GMT

\section{Twitter}

Já foram notificados 162.653 casos suspeitos de \#dengue em todo RJ, com 22 óbitos. Não dê mole pro mosquito! http://t.co/06LTwr3C) casos suspeitos de \#dengue em todo RJ, com 22 óbitos. Não.

SaudeGovRJ

Tue. 14 May 2013 20:33:35 GMT

\section{Fontes Oficiais}

RELATÓRIO DE CASOS DE DENGUE ? 19 / 201 Noticias Saúde RELATÓRIO DE CASOS DE DENGUE? $19 / 2013$

Noticias Secretaria de Estado de Saúde - SES Tue, 14 May 2013 23:35:00 GMT

Agenda Flocruz: 13/5 a1715
Segunda-feira: $13 / 52^{\circ}$ Simpósio Hermann
Schatzmayr Horário: 8 h30 Local: Auditório
Emmanuel..
FIOCRUZ - Fundação Oswaldo Cruz
Mon, 13 May 2013 20:54:21 GMT
Projeto inovador contra a dengue avança no estado
do Rio
Trazido ao Brasil pela Fiocruz em 2012, o projeto
Eliminar a Dengue: Desafio Brasil entra em nova...
FlOCRUZ - Fundação Oswaldo Cruz
Fri, 10 May 2013 20:54:35 GMT

Mais

Blog Archive $\ddot{A} A_{w}$ Bacteria-infected mosquitoes may halt malaria

Zihyong $X i$ and his team from MSU injected

wolbachia bacteria into thousands of mosquito embryos...

Miracle Pregnancy

Sun, 12 May 2013 01:07:34 GMT

Aedes aegypti: quanto mais você conhecê-lo, saber seus hábitos, como ele se comp...

Aedes aegypti: quanto mais vocế connecê-lo, saber seus hábitos, como ele se comporta e de que..

Mural de Rio Contra Dengue no Facebook

Tue, 14 May 2013 22:00:35 cMT

\#Dengue News: Dengue fever cases on rise in
Kottayam - Times of India http://t.co/MIffPel2Ex \#Dengue News: of India hitp:/ftcom Kottayam - Times of India

DengueNews

Tue, 14 May 2013 19:38:04 GMT

Inicial |Sobre o Projeto Monitoramento | Contato
Produçäo Cientifica

Study defines level or dengue virus needed for

(Wellcome Trust) Researchers have identified the dose of dengue virus in human blood that is

EurekAlert - Biology

Mon. 13 May 2013 03:00:00 GMr

Sickly mosquitoes stymle malaria s spread Nature.com3ickly mos quitoes stymie malaria's (1)

Nature.com

Thu, 09 May 2013 21:01:55 GMT

The increase in cholesterol levels at early stages after dengue virus infection correlates with an augment in LDL particle uptake and HMG-COA

reductase activity

Publication date: Available online 2 May 2013Source:Virology Author(s): Rubén...

ScienceDirect Publication: Virology Tue, 07 May 2013 03:34:23 GMT

\section{Fiocruz: nova técnica promete reduzir casos de} dengue I Campos ...

mosquitos Aedes aegypti a Wo entrar no...

Campos 24Horas | Seu Jornal na internet!

Sat. 11 May 2013 18.23.20 GMT

Com a automedicação você pode mascarar os sintomas da dengue e dificultar o diag... Com a automedicação você pode mascarar os sintomas da dengue e dificultar o diagnóstico....

Mural de Combata a Dengue no Facebook Tue, 14 May 2013 20:38:22 CMT

Combate à Dengue é intensificado após registro de casos em Tietê (SP): http://t.co/AuRyvLizoo Combate à Dengue e intensificado após registro de casos em Tietê (SP):

combatadengue

Tue, 14 May 2013 18:16:59 GMT

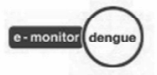

Figura 1. O espaço virtual e-Monitor Dengue.

Fonte: E-monitor Dengue. Disponível em: <http://www.emonitordengue.icict.fiocruz.br>. 
Quadro 1. Relação de perfis no Twitter com maior ocorrência do termo "dengue".

\begin{tabular}{|c|c|}
\hline Fonte oficial & Tweets com o termo dengue $\left(\mathrm{n}^{\circ}\right)$ \\
\hline @RioContraDengue & 244 \\
\hline @GovRJ _ n & 38 \\
\hline$@$ @GovRJ & 38 \\
\hline @SaudeGovRJ & 33 \\
\hline @cms_epol & 31 \\
\hline @cvasrio & 26 \\
\hline @cvasrio & 26 \\
\hline @CAP10_Rio & 13 \\
\hline @oticsbarra & 13 \\
\hline @CAP_51 & 12 \\
\hline @Sesc_Rio & 11 \\
\hline @Rioeduca & 10 \\
\hline @MultiRio & 7 \\
\hline @CanalSaude & 6 \\
\hline @agencia_fiocruz & 5 \\
\hline @ces_rj _ n & 5 \\
\hline @ensp & 4 \\
\hline @OticsRio & 4 \\
\hline @csfmso & 4 \\
\hline @Cap21ZS & 3 \\
\hline @SMSDC_Rio & 3 \\
\hline @CAP_52 & 2 \\
\hline Fonte de notícia & Tweets $\left(n^{\circ}\right)$ \\
\hline @InformeRJO & 39 \\
\hline @correiodobrasil & 25 \\
\hline @rj_noticia & 21 \\
\hline @NiteroiAlerta & 20 \\
\hline @radar_rj & 12 \\
\hline @NitFeed & 11 \\
\hline @bandnewsfmrio & 8 \\
\hline @Niteroi24hrs & 7 \\
\hline @ReporterRio & 7 \\
\hline @JornalOGlobo & 6 \\
\hline @JornaldoBrasil & 5 \\
\hline @JornalExtra & 5 \\
\hline @Encontrarj & 5 \\
\hline @Rionewsnow & 5 \\
\hline @Niteroiempauta & 4 \\
\hline$@$ @Ecodebate & 4 \\
\hline @planetaniteroi & 4 \\
\hline @expresso_fm & 3 \\
\hline @SuperRadioTupi & 2 \\
\hline @RJ_Total & 2 \\
\hline @Rjnoticias & 2 \\
\hline @Niteroinews & 1 \\
\hline @OEstadoRJ & 1 \\
\hline Instituição de ensino & Tweets com o termo dengue $\left(n^{\circ}\right)$ \\
\hline @Unigranrio & 2 \\
\hline Profissionais de saúde & Tweets com o termo dengue $\left(n^{\circ}\right)$ \\
\hline @Blogdoavs & 54 \\
\hline @SINDHRio & 3 \\
\hline
\end{tabular}

Na 39a semana epidemiológica o número de tweets tem um aumento considerável em relação ao número de tweets das semanas anteriores. A partir da mineração de textos (Text Mining) das mensagens monitoradas e armazenadas no banco de dados durante esse período, foi possível identificar que cerca de 40\% das postagens apresentaram a ocorrência do termo Wolbachia. Nesse período foi anunciado por cientistas da Fundação Oswaldo Cruz (2012), durante o $18^{\circ}$ Congresso Internacional de Medicina Tropical e Malária, realizado no Rio de Janeiro, a utilização da Wolbachia para bloquear a transmissão do vírus da dengue pelo mosquito Aedes aegypti. A Wolbachia é um gênero de bactéria intracelular que está presente naturalmente em 70\% (ou na maioria) dos insetos, inclusive em algumas espécies de mosquitos. A notícia foi amplamente lida e comentada, indicando a atenção do público para o tema. Acredita-se que futuramente a partir da exploração dessas mensagens, seja possível o entendimento de como as mídias sociais podem ser mobilizadas para fortalecer a relação ciência-sociedade.

Para verificar a associação estatística entre os casos notificados e o número de twetts foi utilizado o Coeficiente de Correlação de Spearman. O coeficiente $p$ de Spearman varia entre -1 e 1, ou seja, quanto mais próximo estiver destes extremos, maior será a associação entre as variáveis. Observa-se que houve uma correlação significativa entre os casos notificados de dengue entre abril e setembro de 2012 e o número de tweets durante o mesmo período, sendo $r=0,75$ e $p$-valor $<0,001$. Conforme pode ser visto na Figura 3, quanto maior o número de casos notificados, maior é o número de tweets. Pode-se concluir que em tempos de epidemia de dengue, o assunto é mais comentado no Twitter. Dessa forma, a partir de tweets com o termo dengue e os casos notificados por meio de mecanismos oficiais, foi mostrado que as fontes de mídia social se correlacionam fortemente com os dados notificados oficialmente. Vale ressaltar que, para esse estudo, foi considerado apenas o volume de tweets relacionados à dengue, postados por usuários no Rio de Janeiro, durante a 20a e 45a semana epidemiológica.

Um número crescente de estudos vem reafirmando que dados de fontes não oficiais podem ser utilizados para o uso de vigilância sindrômica. Especificamente, em 


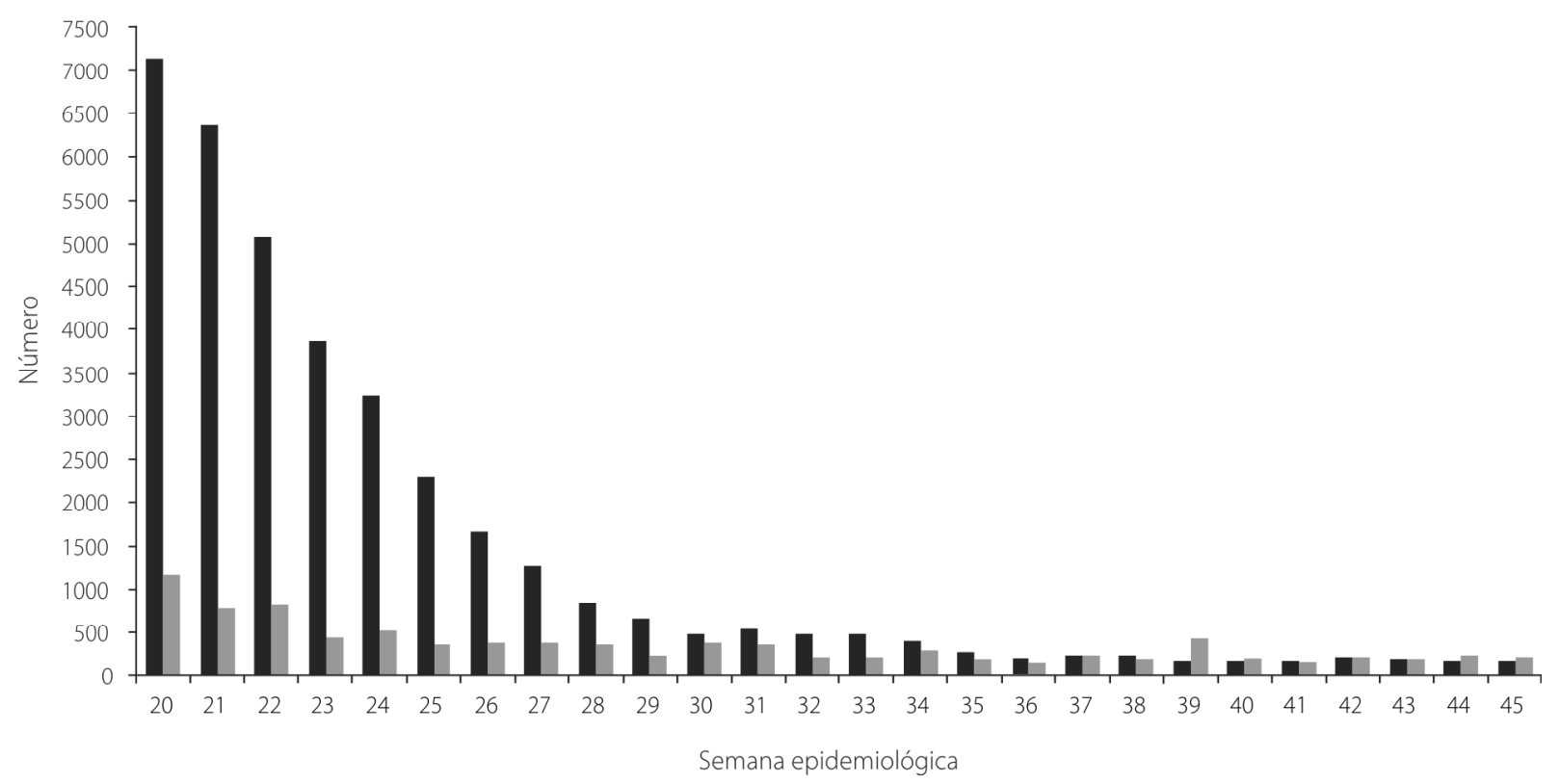

- Número de casos notificados

Número de tweets

Figura 2. Casos notificados por semana epidemiológica x Twitter. Rio de Janeiro (RJ). Semanas epidemiológicas: $20^{a}$ a $45^{a}$.

Fonte: E-monitor Dengue. Disponível em: <http://www.emonitordengue.icict.fiocruz.br>.

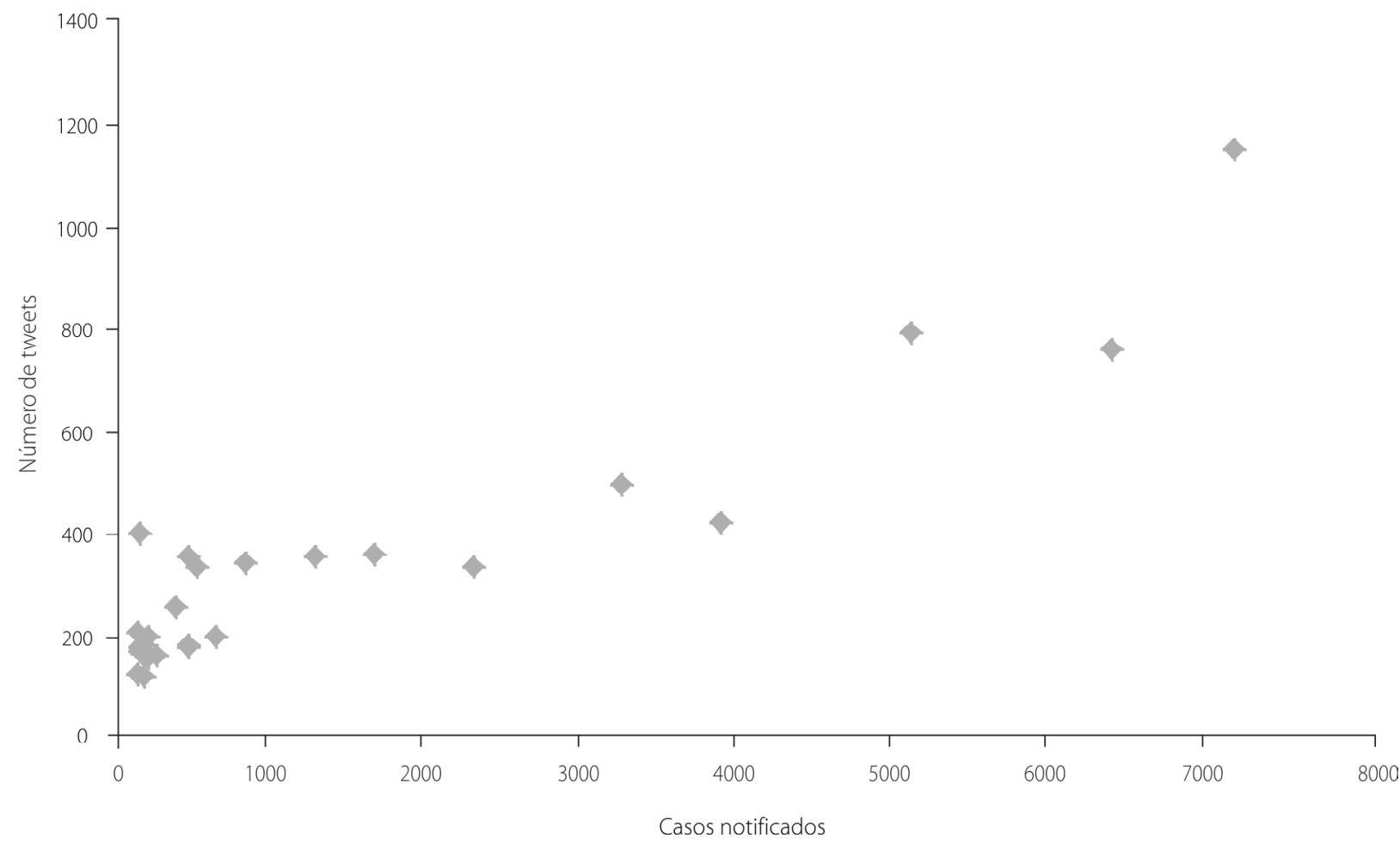

Figura 3. Casos notificados por semana epidemiológica e tweets. Rio de Janeiro (RJ). Semanas epidemiológicas: $20^{a}$ a $45^{a}$.

Fonte: E-monitor Dengue. Disponível em: <http://www.emonitordengue.icict.fiocruz.br>. 
"Dengue surveillance based on a computational model of spatio-temporal locality of Twitter", Gomide et al. (2011) confirmam o potencial de dados do Twitter para a vigilância da dengue. Chunara et al. (2012) concluíram durante a epidemia de cólera no Haiti que o volume de notícias e as postagens no Twitter tiveram também uma forte correlação com os relatórios oficiais.

Eysenbach (2011) discute as vantagens e limitações do uso de dados coletados a partir da Internet, especialmente no campo da saúde pública. De acordo com o autor, uma das principais vantagens está na coleta dos dados que podem ser coletados automaticamente em tempo real e com baixo custo, além de proporcionar análises quantitativas e qualitativas. Mas, ao mesmo tempo, é importante notar certas limitações, como, por exemplo, no caso do Twitter, uma vez que os usuários da Internet que usam as mídias sociais não são representativos de toda a população.

Vale ressaltar que esses métodos não substituem os métodos tradicionais de vigilância, no entanto, os resultados indicam que o twitter pode ser utilizado como uma fonte complementar aos métodos atuais para a vigilância epidemiológica.

\section{Considerações Finais}

O Twitter apresenta-se como uma ferramenta eficaz para a disseminação de informação, e, por isto, ele tem sido adotado maciçamente em muitos setores, incluindo a saúde. Na saúde, o Twitter tem emergido como uma ferramenta promissora para o monitoramento e detecção de epidemias, além de proporcionar indícios para a avaliação de como as mídias sociais são usadas de forma interativa pelas organizações no envolvimento do público nas questões que envolvem a saúde.

Desta forma, o Twitter pode também desempenhar um papel na gestão da informação a partir da possibilidade da identificação dos usuários que podem atuar como filtro de informação, pois assim é possível acessar diretamente a informação mais relevante para uma determinada área de interesse. No caso do e-Monitor Dengue, os perfis identificados, sejam eles oficiais ou noticiosos, são monitorados constantemente.

A partir do monitoramento do Twitter, espera-se que seja possível o entendimento de como as mídias sociais podem ser mobilizadas para fortalecer a relação ciência-sociedade, como ocorrido durante o anúncio pela ciência sobre a utilização de um novo método de controle do mosquito transmissor da dengue. Espera-se ainda que as mídias sociais funcionem mais como um canal que possibilite o entendimento público da ciência pela sociedade, diminuindo o tão propalado "gap" entre cientistas e o público.

Por fim, os resultados obtidos até o presente momento indicam também o potencial do e-Monitor Dengue para a vigilância do ambiente informacional, por meio do rastreamento de rumores sobre epidemias com maior facilidade, proporcionando uma resposta mais rápida para os tomadores de decisão. Evidencia-se, desta forma, que realizar o monitoramento nas mídias sociais durante o período de epidemia e fazer sua relação com a situação epidemiológica da dengue pode ser uma estratégia importante para as autoridades em vigilância epidemiológica.

\section{Referências}

Akshay, J. et al. Why we twitter: Understanding microblogging usage and communities. In: Workshop on Web Mining and Social Network Analysis, 1., 2007, San Jose, California. Proceedings... New York: ACM SIGKDD, 2007. p.56-65.

Brasil. Ministério da Saúde. Ministério lança campanha de combate à dengue. 2011. Disponível em: <http://portalsaude. saude.gov.br/portalsaude/noticia/3563/162/ministerio-lancacampanha-de-combate-a-dengue.html>. Acesso em: 5 dez. 2011.

Chan, E.H. et al. Using web search query data to monitor dengue epidemics: A new model for neglected tropical disease surveillance. PLoS Neglected Tropical Diseases, v.5, n.5, p.1206,

2011. Available from: <http://www.ncbi.nlm.nih.gov/pmc/ articles/PMC3104029/>. Cited: July 22, 2013.

Choo, C.W.; Auster, E. Environmental scanning: Acquisition and use of information by managers. Annual Review of Information Science and Technology, v.28, p.279-281, 1993.

Chunara, R. et al. Social and news media enable estimation of epidemiological patterns early in the 2010 Haitian cholera outbreak. The American Journal of Tropical Medicine and Hygiene, v.86, n.1, p.39-45, 2012. Available from: <http://http:// www.ncbi.nlm.nih.gov/pubmed/22232449>. Cited: July 22, 2013. 
Coates, J. Foresight in federal government policy making: Futures research quartely, 1985. In: Porter, A. et al. Forecasting and management of technology. New York: J. Wiley, 1991. p.29-53.

Eysenbach, G. Infodemiology and infoveillance tracking online health information and cyberbehavior for public health. American Journal of Preventive Medicine, v.40, n.5, p.S154-S158, 2011. Available from: <http://www.ncbi.nlm.nih.gov/ pubmed/21521589>. Cited: June 22, 2013.

Eysenbach, G.; Chew, C. Pandemics in the age of twitter: Content analysis of tweets during the $2009 \mathrm{H} 1 \mathrm{~N} 1$ outbreak. PLOS ONE, v.5, n.11, 2010. Available from: <http://www. plosone.org/article/info\%3Adoi\%2F10.1371\%2Fjournal.pone. 0014118>. Cited: Sept. 15, 2012.

Freifeld, C.C. et al. Participatory epidemiology: Use of mobile phones for community-based health reporting. PLoS Medicine, v.7, 2010. doi: 10.1371/journal.pmed.1000376

Fundação Oswaldo Cruz. Parceria trás para o Brasil iniciativa pioneira de pesquisa em dengue. 2012. Disponível em: <http:// www.fiocruz.br/ccs/cgi/cgilua.exe/sys/start.htm?from info index=121\&infoid=4855\&sid=9>. Acesso em: 24 set. $201 \overline{2}$.

Ginsberg J. et al. Detecting influenza epidemics using search engine query data. Nature, v.457, n.7232, 2009. Available from: <http://static.googleusercontent.com/external_content/ untrusted_dlcp/research.google.com/en//archive/papers/ detecting-influenza-epidemics.pdf>. Cited: July 22, 2013.

Gomide, J. et. al. Dengue surveillance based on a computational model of spatio-temporal locality of Twitter. 2011. In: ACM Web Science Conference, 11., 2011, Koblenz, Germany. Proceedings... Koblenz, Germany: ACM, 2011. Available from: <http://www.websci11.org/fileadmin/websci/ Papers/92_paper.pdf>. Cited: July 22, 2013.

Guimarães, M.C.S.; Silva, C.H.; Antunes, M.N. Monitoramento de informação como estratégia de e-health: um estudo prospectivo. Revista Textos de la CiberSociedad, n.16, 2008. Disponível em: <http://www.cibersociedad.net>. Acesso em: 24 jun. 2011.

Hoffmann, W. Monitoramento da informação e inteligência competitiva: realidade organizacional. InCID: Revista de Ciência da Informação e Documentação, v.2, n.2, 2011. Disponível em: <http://revistas.ffclrp.usp.br/incid/article/view/84>. Acesso em: 22 jul. 2013.

Keckley, P.H. Social networks in health care: Communication, collaboration and insights. Deloitte Center for Health Solutions,
2010. Available from: <http://www.deloitte.com/assets/ Dcom-UnitedStates/Local\%20Assets/Documents/ US_CHS_2010SocialNetworks_070710.pdf>. Cited: June 24, 2011.

Organização Mundial da Saúde. World report on knowledgefor better health. Geneva: World Health Organization, 2009. Available from: <http://www.who.int/rpc/meetings/en/ WR2004AnnotatedOutline.pdf>. Cited: June 24, 2011.

Rio de Janeiro (Município). Secretaria Municipal de Saúde e Defesa Civil. Casos de Dengue por bairro e período. 2012. Disponível em: <http://www.rio.rj.gov.br/web/sms/exibe conteudo?id=2815389 . . Acesso em: 30 dez. 2012.

Santella, L.; Lemos, R. Redes sociais digitais: a cognição conectiva do Twitter. São Paulo: Paulus, 2010.

Scanfeld, D.; Scanfeld, V.; Larson, E.L. Dissemination of health information through social networks: Twiter and antibiotics. American Journal of Infection Control, v.38, p.182-188, 2010.

Silva, C.H. Monitoramento de informação na sociedade de risco: o caso da gripe aviária Rio de Janeiro: Fiocruz, 2007. (Projeto de Pesquisa).

Sterne, J. Métricas em mídias sociais. São Paulo: Nobel, 2011.

Thackeray, R. et al. Adoption and use of social media among public health departments. BMC Public Health, v.12, n.242, 2012.

Torrente, E.; Martí, T., Escarrabill, J. A breath of twitter. Revista Portuguesa de Pneumologia, v.18, n.3, p.137-141, 2012.

Valentim, M.L.P.; Molina, L.G. Prospecção e monitoramento informacional no processo de inteligência competitiva. Encontros Bibli, n. esp., p.59-77, 2004. Disponível em: <http:// www.journal.ufsc.br/index.php/eb/article/viewArticle/292>. Acesso em: 24 jun. 2011.

Vance, K.; Howe, W.; Dellavalle, R.P. Social internet sites as a source of public health information. Dermatologic Clinics, v.27, n.2, p.133-136, 2009.

Wang, P.et al. Understanding the spreading patterns of mobile phone viruses. Science, v.324, p.1071-1076, 2009.

Yan, P.; Chen, H.; Zeng, D. Syndromic surveillance systems. Annual Review of Information Science \& Technology, v.42, p.425-495, 2009. Available from: <http://www.sciencemag. org/content/324/5930/1071>. Cited: July 22, 2013. 
